BULLETIN OF THE

AMERICAN MATHEMATICAL SOCIETY

Volume 80, Number 1, January 1974

\title{
QUASI-KAN EXTENSIONS FOR 2-CATEGORIES
}

\author{
BY JOHN W. GRAY ${ }^{1}$
}

Communicated by Saunders Mac Lane, May 21, 1973

1. Introduction. Let $\mathscr{C}$ at denote the category of small categories and functors. $\mathscr{C} a t$ is a Cartesian closed category, [2] and the prefix 2will denote categories and functors enriched in $\mathscr{C} a t$. 2-שat denotes the category of small 2-categories and 2-functors. It is also Cartesian closed, but there is another notion of a transformation between 2-functors $F$ and $G$ which has interesting properties; namely a quasi-natural transformation from $F$ to $G$ is a family of morphisms $\left\{\varphi_{A}: F(A) \rightarrow G(A)\right\}$ together with a family of 2-cells $\left\{\varphi_{f}: G(f) \varphi_{A} \rightarrow \varphi_{B} F(f)\right\}$ as illustrated

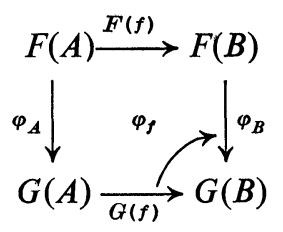

satisfying obvious compatibility conditions. (The case where the $\varphi_{f}$ 's are isomorphisms has been considered in [7] and [8], but we make no such restriction.) Given this notion of "natural transformation", it is reasonable and useful to inquire about the corresponding notion of "quasi-limit" or, more generally, "quasi-Kan extension".

Such a Kan extension was used in an essential way for the proof of the main result in $[4, \S 9]$, but until now no justification has been given for calling the construction used there a "Kan extension". In the usual case, if $S: \mathscr{A} \rightarrow \mathscr{B}$ is an ordinary functor and $\mathscr{X}$ is a cocomplete category, then under appropriate hypotheses the functor

$$
\mathscr{X}^{S}: \mathscr{X}^{B} \rightarrow \mathscr{X}^{A}
$$

is right adjoint to the (left) Kan extension $\Sigma S: \mathscr{X}^{A} \rightarrow \mathscr{X}^{B} . \Sigma S$ can be constructed as follows: replace $S$ by its associated factorization through an opfibration

$$
\mathscr{A} \underset{P}{\stackrel{Q_{s}}{\rightleftarrows}}(S, \mathscr{B}) \stackrel{P_{s}}{\longrightarrow} \mathscr{B}
$$

AMS (MOS) subject classifications (1970). Primary 18D05, 18A40; Secondary 18D25, 18D30.

${ }^{1}$ Supported by NSF Grant GP 33143. 
where $S=P_{S} Q_{S}$ and $P$ is left inverse, right adjoint to $Q_{S}$ (see [3, p. 55]). One shows that for the opfibration $P_{S}$, the Kan extension $\Sigma P_{S}$ is given by "integration (i.e., colimit) along the fibres" and then that $\Sigma S=\left(\Sigma P_{S}\right) \mathscr{X}^{P}$.

In the 2-category case, 2-functors and quasi-natural transformations are the objects and morphisms of a 2-category $\operatorname{Fun}(\mathscr{A}, \mathscr{B})$ which is the internal hom object for a nonsymmetrical, monoidal closed structure

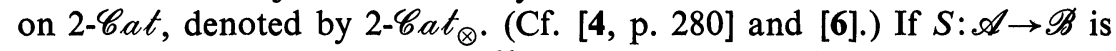
a fixed 2 -functor, then for any $\mathscr{X}$, there is an induced functor

$$
S^{*}=\operatorname{Fun}(S, \mathscr{X}): \operatorname{Fun}(\mathscr{B}, \mathscr{X}) \rightarrow \operatorname{Fun}(\mathscr{A}, \mathscr{X})
$$

and we can ask for a left quasi-adjoint $\Sigma_{q} S$ to $S^{*}$ in some suitable sense of quasi-adjointness. (The case $\mathscr{B}=1$ yields quasi-colimits.) In this paper we describe how to modify each step in the procedure described above to fit the situation of 2-categories. It will be seen that when $S$ is a suitable kind of quasi-opfibration, one obtains an ordinary $\mathscr{C} a t$-enriched adjoint (= $\mathscr{C} a t$-adjoint). This includes the case of quasi-limits. However, in general one gets a strict quasi-adjoint. This notion forces itself upon one when one studies $2-\mathscr{C} a t_{\otimes}$ seriously, since it arises in many different contexts. (In particular, the comprehension scheme in [4] is a strict quasi-adjoint, as are all the constructions mentioned in this paper.) Detailed proofs will be published in [6].

2. Definitions. Besides the 2-comma category $\left[S_{1}, S_{2}\right]$ defined as in [4, p. 279], for a pair of 2-functors $S_{i}: \mathscr{A}_{i} \rightarrow \mathscr{B}$, there are 3-comma categories $\left[S_{1}, S_{2}\right]_{3}$ and $\left[S_{1}, S_{2}\right]_{\otimes}$ defined for 3-functors (resp. 2-C̈at $t_{\otimes^{-}}$ functors) $S_{1}$ and $S_{2}$ between 3-categories (resp., 2-C a $t_{\otimes}$-categories) with the same codomain. 0 -cells and 1-cells are defined as in $\left[S_{1}, S_{2}\right], 2$-cells are a pair of 2-cells as in $\left[S_{1}, S_{2}\right]$ plus a 3-cell expressing the lack of commutativity, and 3-cells are pairs satisfying the obvious equation. Details will be given in [6].

Let $F: \mathscr{A} \rightarrow \mathscr{B}$ and $U: \mathscr{B} \rightarrow \mathscr{A}$ be 2-functors between 2-categories. A pair of quasi-natural transformations, $\varepsilon: F U \rightarrow \mathscr{B}$ and $\eta: \mathscr{A} \rightarrow U F$ is called a quasi-adjunction between $F$ and $U$ if it satisfies the usual equations. It is called strict if

$$
(U \varepsilon F)\left(\eta_{\eta}\right)=1_{\eta}, \quad \varepsilon_{\varepsilon}(F \eta U)=1_{\varepsilon} .
$$

Here, for instance, since $\eta_{A}: A \rightarrow U F A$ is an arrow, $\eta$ assigns to it a 2-cell from $\left(U F \eta_{A}\right) \eta_{A}$ to $\left(\eta U F_{A}\right) \eta_{A}$. This defines the modification (2-cell in Fun $(\mathscr{A}, \mathscr{A})$ ) denoted by $\eta_{\eta}$. Similarly, $1_{\eta}$ is the identity modification of $\eta$. 
3. Cartesian quasi-limits and colimits. In the special case of induced functors $S^{*}$ in which $\mathscr{B}=\mathbf{1}$, one obtains the constant embedding

$$
\Delta_{\mathscr{X}}: \mathscr{X} \rightarrow \operatorname{Fun}(\mathscr{A}, \mathscr{X}) \text {. }
$$

The right (resp. left) $\mathscr{C} a t$-adjoint to $\Delta_{\mathscr{X}}$ is called the Cartesian quasi-limit (resp., colimit) of type $\mathscr{A}$ in $\mathscr{X}$ and is denoted by

$$
\underset{\mathscr{Q}}{Q_{\mathscr{A}}} \overline{\mathscr{C} a t}\left|\Delta_{\mathscr{X}} \frac{}{\mathscr{C} a t}\right| Q_{\mathscr{A}}
$$

$\mathscr{X}$ is called Cartesian quasi-complete (resp., cocomplete) if $Q_{\mathscr{A}}$ (resp., $Q_{\mathscr{A}}$ ) exists in $\mathscr{X}$ for all small 2 -categories $\mathscr{A}$.

TheOREM 1, Cat is Cartesian quasi-complete and cocomplete.

Proof. We describe the construction here.

Let $H: \mathscr{A} \rightarrow \mathscr{C}$ at be a 2 -functor. $H$ determines an opfibration $P: \mathscr{E}_{H} \rightarrow \mathscr{A}$ (see [4, pp. 248, 285, 289]) where $\mathscr{E}_{H}$ is a 2-category. In the special case that $H: \mathscr{A} \rightarrow \mathscr{S}$ ets with $\mathscr{A}$ an ordinary category, then $\lim _{\rightarrow} H=\pi_{0}\left(\mathscr{E}_{H}\right)$ and $\lim H=\Gamma\left(\mathscr{E}_{H}\right)$ where $\pi_{0}$ assigns to a category its set of connected components, and $\Gamma$ is the set of sections of $P$. In the general case one shows that $Q H=L \pi_{0}\left(\mathscr{E}_{H}\right)$ and $Q H=\Gamma\left(\mathscr{E}_{H}\right)$ where $L \pi_{0}: 2-\mathscr{C} a t \rightarrow \mathscr{C}$ at is "local $\pi_{0}$ ", $\overrightarrow{\text { i.e., }}$ it turns a 2-category $\mathscr{C}$ into a category $L \pi_{0}(\mathscr{C})$ by replacing each hom-category $\mathscr{C}(X, Y)$ by the set $\pi_{0} \mathscr{C}(X, Y)$. (Note that this differs from the assertion in [4, p. 289].) Similarly, $\Gamma$ denotes the category of sections of $P$; i.e., 2-functors $G: A \rightarrow \mathscr{E}_{H}$ such that $P G=1$ and natural transformations $\psi$ such that $P \psi=1$.

In [5] it is asserted, and it will be proved elsewhere, that this result holds for strongly representable (resp., corepresentable) 2-categories. These are essentially 2-categories which are complete (resp., cocomplete) in the sense of closed categories.

We list here a number of examples of Cartesian quasi-limits and colimits in $\mathscr{C}$ at. These examples serve to define the corresponding concepts in other 2-categories.

(i) $\mathscr{A}=2$ (the category with two objects and a single nonidentity morphism). $H: \mathbf{2} \rightarrow \mathscr{C}$ at looks like a functor $f: A \rightarrow B$ between small categories and $Q H=(f, B)$, the universal opfibration associated to $f$, while $Q H=\langle A, \vec{f}\rangle$, the universal cofibration associated to $f$ (see $[3, \S 5])$.

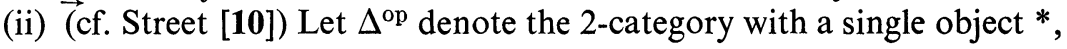
with $\operatorname{Hom}(*, *)$ the dual of the category of finite ordinals. A 2-functor $H: \Delta^{\mathrm{op}} \rightarrow \mathscr{C}$ at is the same as a small category $\mathscr{A}$ equipped with a cotriple $G$, and $Q H$ is the co-Kleisli category of the cotriple. Let ${ }^{\text {op }}\left(\Delta^{\mathrm{op}}\right)$ be the weak dual. Then $H:{ }^{\mathrm{op}}\left(\Delta^{\mathrm{op}}\right) \rightarrow \mathscr{C}$ at is a small category equipped with a triple and $Q H$ is the category of Eilenberg-Moore algebras. Appropriate duals give the other two possibilities. 
If the possibilities are extended by allowing nonfull subcategories of Fun $(\mathscr{A}, \mathscr{B})$ determined by imposing conditions on the 2-cells $\varphi_{f}$ for certain $f$ 's, then $\mathscr{C}$ at still admits such quasi-limits and colimits. As particular examples, one obtains comma categories [3] and subequalizers (Lambek [9]) as well as the result that the closure of $\mathscr{S}$ et $s \subset \mathscr{C}$ at under such quasi-colimits is all of $\mathscr{C}$ at. ing.

The main result about $Q$ needed for quasi-Kan extensions is the follow-

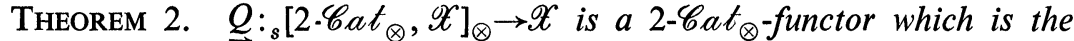
left $2-\mathscr{C} a t_{\otimes}$-adjoint to $N$.

Here $N$ is the functor in the other direction which is the name functor; e.g., on an object $X \in \mathscr{X}, N(X)=X: 1 \rightarrow \mathscr{X}$, etc., and $s$ means small. The main (and considerable) difficulty is to show that $\underset{Q}{\rightarrow}$ is defined here.

4. Quasi-fibrations. Among the various possible definitions the following is the one needed here. Let $\operatorname{Fun}(B)={ }^{\mathrm{op}} \operatorname{Fun}\left(2,{ }^{\text {op }} \mathscr{B}\right) . A$ 2-functor $P: \mathscr{E} \rightarrow \mathscr{B}$ is called a Cartesian quasi-opfibration if there exists a 2-functor $L$ as illustrated

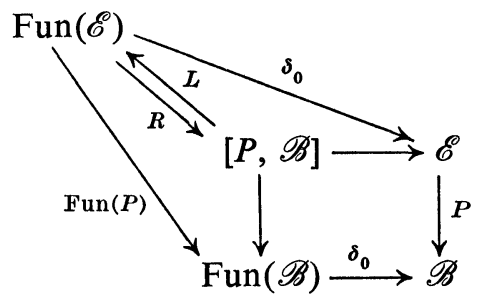

having $R$ as a right $\mathscr{C}$ at-adjoint and $R L=\mathrm{id}$. Here the square is a pullback and $\delta_{0}, \operatorname{Fun}(P)$ and $R$ are the obvious induced 2 functors. A choice of $L$ is called a cleavage. If $L$ is chosen so that $L(\mathrm{id})=\mathrm{id}$ and

$$
L\left(f_{*} E, g\right) \circ L(E, f)=L(E, g f)
$$

then $P$ together with $L$ is called a split-normal Cartesian quasi-opfibration. The 2-category of such together with cleavage preserving 2-functors and $\mathscr{C}$ at natural transformations over $\mathscr{B}$ is denoted by $\operatorname{Cart} q-\operatorname{Split}(\mathscr{B})_{0}$.

THEOREM 3. The inclusion

$$
\text { Cart } q \text {-Split }(\mathscr{B})_{0} \rightarrow\left[{ }^{\mathrm{op}} 2 \mathscr{C} \text { at, } \mathscr{B}\right]
$$

has a strict left quasi-adjoint $\Phi$.

Here $\Phi$ on an object $S: \mathscr{A} \rightarrow \mathscr{B}$ is the projection $P_{S}:[S, \mathscr{B}] \rightarrow \mathscr{B}$. 
THEOREM 4. The associated opfibration has the property that there is a factorization of $S$,

$$
\mathscr{A} \underset{P}{\stackrel{Q_{S}}{\rightleftarrows}}[S, \mathscr{B}] \stackrel{P_{S}}{\longrightarrow} \mathscr{B}
$$

in which $P_{S} Q_{S}=S$ and $P$ is left inverse, strict quasi-right adjoint to $Q_{S}$.

REMARK. $\quad P_{S}$ is also an ordinary $\mathscr{C}$ at-enriched opfibration and $P$ is a Cat-enriched fibration.

THEOREM 5. Let $(P: \mathscr{E} \rightarrow \mathscr{B}, L)$ be a split normal Cartesian quasi-

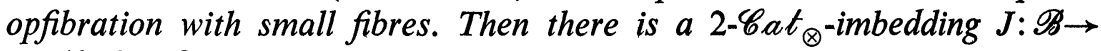
$s\left[2-\mathscr{C} a t_{\otimes}, \mathscr{E}\right]_{\otimes}$.

5. Quasi-Kan extensions. For quasi-opfibrations, one has the following astonishing result.

THEOREM 6. If $P: \mathscr{E} \rightarrow \mathscr{B}$ is a split normal Cartesian quasi-opfibration with small fibres, and $\mathscr{X}$ is Cartesian quasi-cocomplete then $P^{*}: \operatorname{Fun}(\mathscr{B}, \mathscr{X}) \rightarrow$ $\operatorname{Fun}(\mathscr{E}, \mathscr{X})$ has a left $\mathscr{C}$ at-adjoint, $\Sigma_{\alpha} P$ given by "integration along the fibres."

This means that if $G: \mathscr{E} \rightarrow \mathscr{X}$ is a 2-functor, then $\Sigma_{\alpha} P(G)$ is the composition

$$
\mathscr{B} \stackrel{J}{\longrightarrow}\left[2-\mathscr{C} a t_{\otimes}, \mathscr{E}\right]_{\otimes} \stackrel{G_{*}}{\longrightarrow}{ }_{s}\left[2-\mathscr{C} a t_{\otimes}, \mathscr{X}\right]_{\otimes} \stackrel{\stackrel{Q}{\longrightarrow}}{\longrightarrow} \mathscr{X}
$$

where $G_{*}$ denotes composition with $G$.

Finally, we get the desired generalization of Kan extensions.

THEOREM 7. Let $S: \mathscr{A} \rightarrow \mathscr{B}$ be a 2-functor between small 2-categories and let $\mathscr{X}$ be Cartesian quasi-cocomplete. Let $\Sigma_{q} S=\left(\Sigma_{q} P_{S}\right) P^{*}$ where $P_{S}$ and $P$ are as in Theorem 4 . Then ${ }^{\mathrm{op}}\left(\Sigma_{Q} S\right)$ is a strict quasi-left-adjoint to ${ }^{\mathrm{op}}\left(S^{*}\right)$ : ${ }^{\text {op }} \operatorname{Fun}(\mathscr{B}, \mathscr{X}) \rightarrow{ }^{\mathrm{op}} \mathrm{Fun}(\mathscr{A}, \mathscr{X})$.

The claim in [4, \$9], about $\Sigma_{q} S$ is incorrect and the adjunction is in the sense stated here. Part of the reason for the failure of $\Sigma_{q} S$ to be a $\mathscr{C}$ atadjoint in general is that $P$ is transversal to the fibres in $[S, \mathscr{B}]$ which has the effect that $\Sigma_{q} S$ applied to a quasi-natural transformation yields a $\mathscr{C}$ at-natural transformation. An example of $\Sigma_{q} S$ is given in [4]. Others will be given elsewhere in the subject of 2-theories.

\section{REFERENCES}

1. M. C. Bunge, Bifibration induced adjoint pairs, Reports of the Midwest Category Seminar, V (Zürich, 1970), Lecture Notes in Math., vol. 195, Springer, Berlin, 1971, pp. 70-122. MR 45 \#1986.

2. S. Eilenberg and G. M. Kelly, Closed categories, Proc. Conf. Categorical Algebra (La Jolla, Calif., 1965), Springer, New York, 1966, pp. 421-562. MR 37 \#1432. 
3. J. W. Gray, Fibred and cofibred categories, Proc. Conf. Categorical Algebra (La Jolla, Calif., 1965), Springer, New York, 1966, pp. 21-83. MR 35 \#4277.

4. - The categorical comprehension scheme, Category Theory, Homology Theory and their Applications, III (Battelle Institute Conference, Seattle, Wash., 1968, Vol. Three), Springer, Berlin, 1969, pp. 242-312. MR 40 \#2728.

5. - Reports of the Midwest category seminar, Lecture Notes in Math., vol. 195, Springer-Verlag, Berlin and New York, 1971, pp. 248-255. MR 43 \#4873.

6. - Formal category theory-Adjointness for 2 categories, Lecture Notes in Math., Springer-Verlag, New York (to appear).

7. J. Giraud, Cohomologie non abelienne, Springer-Verlag, New York, 1971.

8. M. Hakim, Topos anneles et schemas relatifs, Springer-Verlag, New York, 1972.

9. J. Lambek, Subequalizers, Canad. Math. Bull. 13 (1970), 337-349. MR 43 \#315.

10. R. Street, The formal theory of monoids, J. Pure Appl. Algebra 2 (1972), 149-168.

Department of Mathematics, University of Illinois at Urbana-Champaign, URBANA, ILLINOIS 61801 